\title{
Gewinner AOTD-Nachwuchsförderung
}

Richard Stange

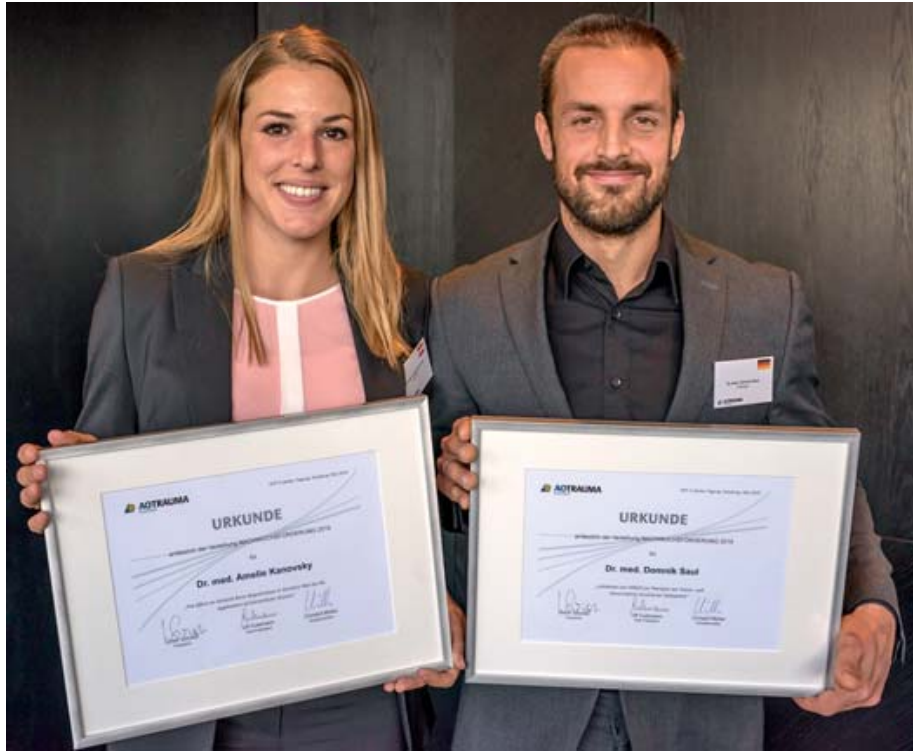

- Abb. 1 Gewinner Kanovsky und Saul. Quelle: AO Foundation

Auf der AOTrauma 3-Länder-Tagung 2019 in Hamburg wurde zum 7. Mal die AOTD-Nachwuchsförderung vergeben. Wegen der gemeinsamen Tagung durften zum ersten Mal auch Ärztinnen und Ärzte aus Österreich und der Schweiz teilnehmen. Aus 14 Bewerbungen wählte die Auswahlkommission, bestehend aus Prof. Georg Duda,
Prof. Hans-Christoph Pape und Prof. Richard Stange, 1 Kandidatin und 3 Kandidaten aus, die auf der Preisträgersitzung ihre Forschungsvorhaben vorstellen durften. Die Preisträgersitzung leiteten Hans-Christoph Pape, Richard Stange und Dr. Michele Arigoni aus der Schweiz. Die Entscheidung fällten die anwesenden Mitglieder per geheime Abstimmung.

Die Förderung in Höhe von jeweils 15000 EUR erhielten Frau Dr. Amelie Kanovsky aus Klagenfurt, Österreich, für das Vorhaben The Effect on Femoral Bone Regeneration in Geriatric Rats by the Application of Extracellular Vesicles und Dr. Dominik Saul, Göttingen, Deutschland, für Inhibition von FOXO3 zur Therapie von Tumor- und Denervationsassoziierter Sarkopenie.

Dr. Mohamed Omar, Hannover, und Dr. Thomas Rosteius, Bochum, erhielten jeweils ein neues AO Manual und werden in das Mentoring-Netzwerk der AOTD aufgenommen.

Prof. Dr. Richard Stange, Münster

E-Mail: richard.stange@ukmuenster.de

Bibliografie

DOI https://doi.org/10.1055/a-0951-4745

OP-JOURNAL 2019; 35: 198 @ Georg Thieme Verlag KG Stuttgart · New York ISSN 0178-1715 